\title{
Combined transnasal transcervical robotic dissection of posterior skull base: feasibility in a cadaveric model*
}

\author{
lacopo Dallan', Paolo Castelnuovo', Veronica Seccia², Paolo Battaglia', \\ Filippo Montevecchi ${ }^{3}$, Manfred Tschabitscher ${ }^{4, \#}$, Claudio Vicini ${ }^{3, \#}$ \\ Otorhinolaryngologic Unit, University of Insubria, Italy \\ ENT Unit, Azienda Ospedaliero Universitaria Pisana, Pisa, Italy \\ 3. Department of Special Surgery, ENT and Oral Surgery Unit, Ospedale Morgagni Pietrantoni, - University of Pavia in Forli, Italy \\ 4. Department of Systematic Anatomy, University of Wien, Wien, Austria
}

Rhinology 50: 165-170, 2012

DOI:10.4193/Rhino11.079

*Received for publication:

April 14, 2011

accepted: September 14, 2011

\section{Summary}

The current surgical trend is to expand the variety of minimally invasive approaches and, in particular, the possible application of robotic surgery in head and neck surgery. For this purpose, we explored the feasibility of a combined transcervicaltransnasal approach to the posterior skull base, using the da Vinci Surgical System in 3 cadaver heads. Superb visualization of the sellar, suprasellar and clival regions was possible in all three specimens. The trocars' placement through a transcervical port made a more cephalad visualization possible, eliminating the need to split the palate. The advantages of robotic surgery applied to the posterior cranial fossa are similar to the ones already clinically experienced in other districts (oropharynx, tongue base), in terms of tremor-free, bimanual, precise dissection. The implementation of instruments for bony work will definitely increase the applicability of such a system in the forthcoming years.

Key words: robotic surgery, skull base, transcervical surgery, transnasal surgery, combined transcervical-transnasal approach

\section{Introduction}

Skull base procedures are challenging. Traditional external approaches are demanding for both the patient and surgeon. There has been a clear trend during recent years towards minimally invasive solutions. Nowadays, endoscopic transnasal techniques represent a valid alternative to a traditional external approach and probably the gold standard in selected cases of skull base pathologies, especially when dealing with clival and sellarparasellar lesions ${ }^{(1-6)}$. Furthermore, a growing interest in new technologies is evident in recent literature. From the pioneering work by the Philadelphia group, robotic techniques have been applied to different areas outside the tongue base and oropharynx. At the moment, clinical series regarding ventral skull base lesion management are absent with the unique exception of a blended solution on cranio-cervical junction ${ }^{(7)}$. A small series on parapharyngeal lesion management has been reported by the Penn's group ${ }^{(8)}$. More consistent is the preclinical literature on this topic ${ }^{(9-13)}$. These articles demonstrate that there is a growing 'robotic' interest in skull base regions.

In this paper, we report our experience in robotic skull base dissection by means of a combined transnasal-transcervical approach. A review of the pertinent literature, with a focus on advantages, limits and expectations is given.

\section{Materials and methods}

The DaVinci Surgical system (Intuitive", Sunnyvale, San Francisco, (A, USA) was used in one non-injected cadaver and two injected isolated heads. Based on a previous experience ${ }^{(14)}$, we placed trocars for robotic arms and optic lens transcervically and transnasally, respectively. Detailed description of the setting has been given elsewhere ${ }^{(14)}$ and this solution can be considered a modification of the setting described by others for a midline skull base approach ${ }^{(12)}$. From a technical viewpoint, the placement of transcervical paramandibular trocars is done by performing a small paramandibular incision $(<5 \mathrm{~mm})$, close to the angle of the mandible and then reaching, by means of a blunt short dissection, the mandible itself. At this point, the level of the floor of the mouth is reached by a subperiosteal dissection, conducted for about $1 \mathrm{~cm}$, and, by opening the mucosa, the oral cavity space is gained. The whole procedure is performed under direct 

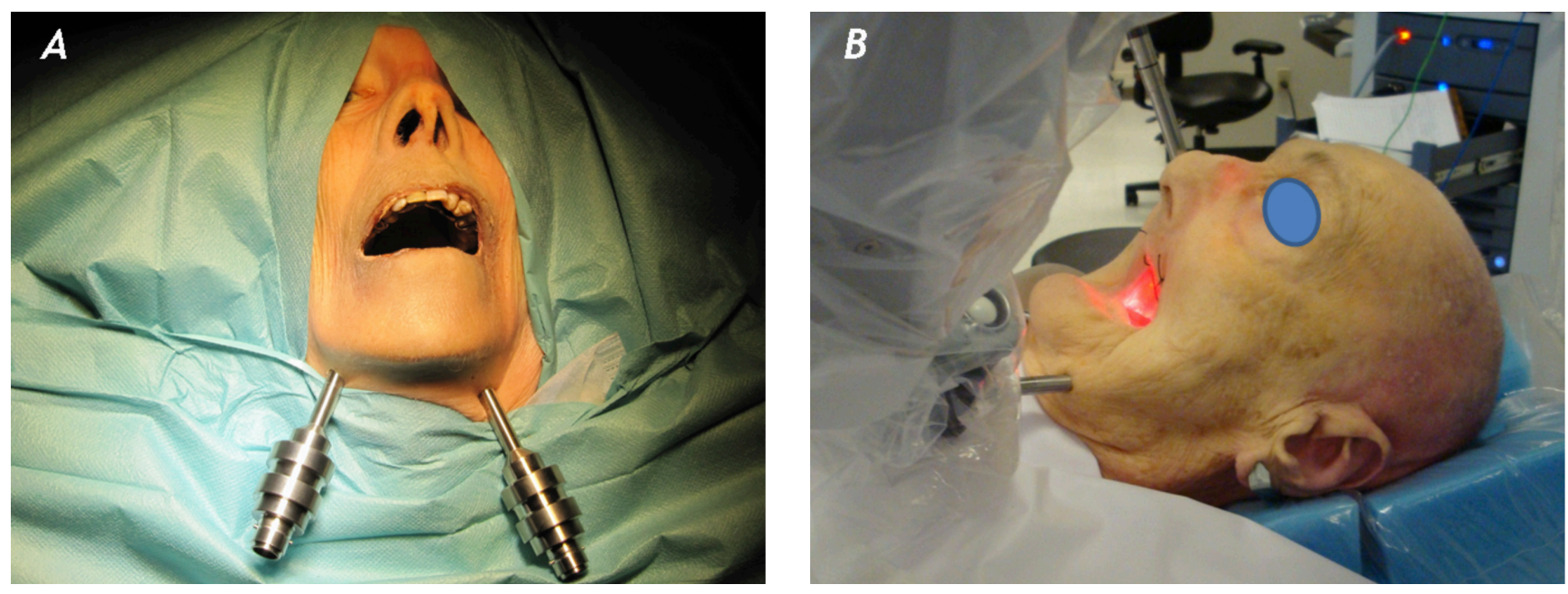

Figure 1. External views (A-frontal, B-lateral) of the setting used. The trocars are inserted behind the mandibular gland, in close relationship to the mandibular angle. The optic lens passes transnasally.

digital control. So, technically, the trocars exit at the level of the posterior floor of the mouth and then can be directed through the oropharynx towards the rhinopharynx. At this point, the trocars are aligned with the robotic end effector instrument along the long axis of the spine and oriented cephalad.

First, a traditional posterior septectomy was performed in a traditional way to facilitate and improve the visualization of the operative field. No palatal splitting was necessary for robotic dissections.

The first part of the dissection was performed by a robotic technique until the bony structures of the skull base were exposed. These structures were removed by traditional transnasal techniques until the dura of the posterior cranial fossa (PCF) and pituitary region was exposed. In this preclinical setting, the bony work was done by drill and cutting instruments. Then the robotic work restarted and dissection of the PCF and pituitary regions was performed. Ability to work and dissect, gentle handling of the vessels and nerves and criticisms during dissection have been subjectively evaluated by the investigators (PC and ID). The length of dissection was not evaluated since the primary outcome of the study was to evaluate the feasibility of the procedure.

At the end of the procedure a palatal splitting was performed to obtain a 'traditional' transoral robotic vision and compare different perspectives.

\section{Results}

Access and dissection of the posterior and central part of the ventral skull base was possible in all specimens. With the transnasal placement of the lens $\left(0^{\circ}\right.$ and $\left.30^{\circ}\right)$, a superb vision of the sellar, suprasellar and clival regions was achieved (Figure 2). The optic chiasm was visible as well. A wide visualization of the pons was obtained and also the abducens nerves were visualized (Figure 3). A complete pituitary transposition was performed (Figure 4) and the basilar tip region was well exposed (Figure 4). Oculomotor nerves passing between the posterior cerebral artery and superior cerebellar artery were visible, as well as the posterior portion of the posterior communicating artery (Figure 5). Detailed vision on the pituitary vascularization was obtained. More in detail, the meningohypophyseal trunk and the superior hypophyseal artery and its arborization, including chiasmal branches, were clearly identified and dissected (Figure 6).

By comparing a transnasal and a transoral perspective, the difference becomes evident. With the transoral placement of the optic, the vision of the chiasmatic region was extremely difficult, as was the pituitary gland. In our hands, the ability to work with a pure transoral approach was really limited.

With a transnasal placement of the optic system, the sellar region can be easily visualized with a $0^{\circ}$ scope, by simply turning the optic lens upwards towards the pituitary region. The use of a $30^{\circ}$ lens allows a more panoramic and comprehensive view of the operative field.

Dissection ability is good. In more cranial regions, we observed a sporadic conflict between the robotic arms. Conflict between instruments was almost absent.

\section{Discussion}

Skull base approaches are challenging. Improvement in anatomical knowledge and surgical technologies offers new solutions and pose different questions. At present, clival, sellar and parasellar region are usually addressed by means of endoscopic 


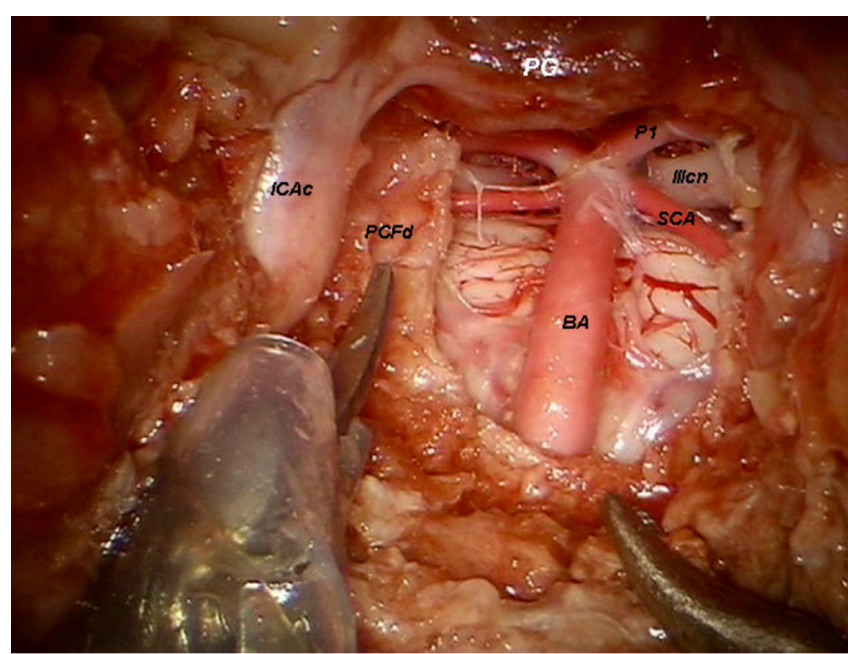

Figure 2. Panoramic view of the mid-upper clivus and pituitary region (10mm, $0^{\circ}$ scope). PG-pituitary gland, PCFd-posterior cranial fossa dura, ICAc-cavernous portion of the internal carotid artery, BA-basilar artery, SCA-superior cerebellar artery, P1-first segment of the posterior cerebral artery, Illcn-oculomotor nerve.

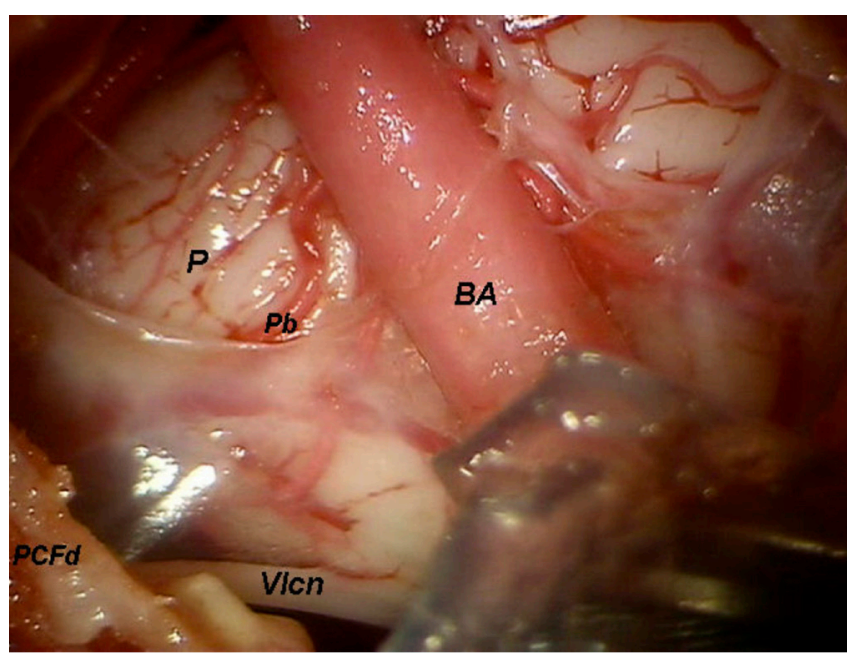

Figure 3. Close view of the right mid clivus, the cisternal part of the abducens nerve is visible. Vision obtained using a $10 \mathrm{~mm}, 30^{\circ}$, turned to the right side. PCFd-posterior cranial fossa dura, BA-basilar artery, VIcnabducens nerve, P-pons, Pb-pontine branch. transnasal procedures. The experience gained in this field, thanks to the work of different groups all around the world (1-6), is nowadays significant. From a technical viewpoint, it has been possible to manage these complex areas thanks to the incredible improvement of the reconstructive techniques ${ }^{(15-20)}$; in this respect the local pedicle flaps have really represented a critical breakthrough event in endoscopic skull base reconstruction ${ }^{(15)}$. In this sense, the pedicle of the naso-septal flap should be spared when approaching the middle and posterior ventral skull base, Very recently, Kupferman et al., have directly addressed the problem of a purely robotic reconstruction of the posterior cranial fossa ${ }^{(21)}$. Without doubt, an ideal surgical technique would offer the distinct advantages of $3 \mathrm{D}$ vision and bimanual surgical dissection ${ }^{(11)}$, possibly guided by a navigation system. In this sense, the da Vinci surgical system seems to guarantee to the surgeon both these opportunities, at least in some anatomical regions. Control at depth in a narrow space is another advantage of the da Vinci system. In this sense, we underline how the "wisted" dissectors also permit the robotic arms to make acute angles that may exceed anatomic roadblocks. The idea to deal with the skull base by means of a robotic technique has been pioneered by MD Anderson's ${ }^{(9)}$ and Penn's ${ }^{(10)}$ groups. Thanks to their work and solutions, a robotic road to the skull base has been opened. In our opinion, although both proposals offer really interesting ideas, at the same time they present some aspects that can be improved. So, based on an extended experience in endoscopic transnasal skull base procedures ${ }^{(22)}$ and in transoral robotic procedures as well (actually $>120$ clinical cases ${ }^{(23)}$ ), we tried to combine the advantage of placing the endoscope transnasally with the advantage of allowing the robotic arms to pass transcervically. We confirm, as previously stated, that the retromandibular placement of the trocars, allows an excellent approach to the upper clivus and pituitary regions ${ }^{(10)}$. But, in particular, we feel that the most significant advantage in our setting is to offer a really familiar and panoramic view of the surgical field. We are strongly convinced that a down-to-up vision makes working ability less comfortable and then less safe in the upper regions. With the transnasal placement of the scope, we obtain a magnificent vision of the entire rhinopharynx and sphenoidal regions, even with a $0^{\circ}$ scope. Furthermore, using a $30^{\circ}$ scope, it is possible to perfectly visualize the region of the tuberculum sellae and caudally the inferior clivus and $\mathrm{C} 1$. Our preclinical experience demonstrates that, by means of a purely transoral approach, the upper work (above the level of the midclivus) is performed at the border of the surgical field. In this setting, with a $30^{\circ}$ upward facing lens, it is possible to visualize the pituitary region but the vision is really unfamiliar to most surgeons. With the current instrumentation, a conflict between the scope and the piriform aperture can be present during the surgical nasal time. We underline that our dissection was performed with a $10 \mathrm{~mm}$ scope which fills the piriform aperture almost completely. Notwithstanding, we maintain that this is a false problem, for two main reasons. First, improvement in technology will offer smaller scopes in the future (nowadays scopes of $8.5 \mathrm{~mm}$ are available) and secondly, if necessary, the piriform aperture can be easily enlarged transnasally (as in a 'Sturmann-Canfield' operation). In the posterior $2 / 3$ rd of the nasal fossa, the conflict is less evident (if not absent) because the realization of a posterior septectomy allows a greater free- 

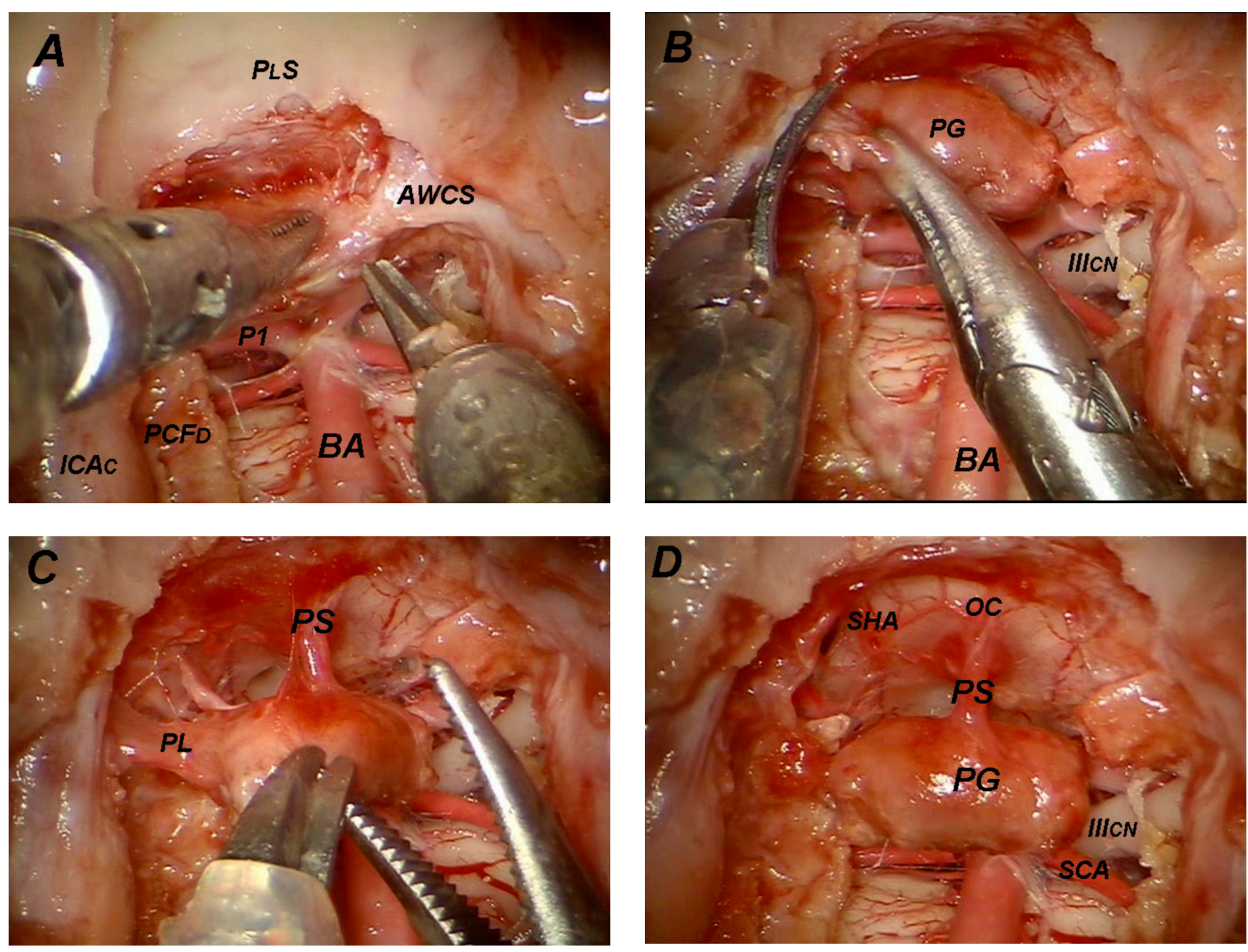

Figure 4. A-D Step by step pituitary transposition. Vision obtained using a $10 \mathrm{~mm}, 30^{\circ}$ scope, upward faced. PLS-planum sphenoidale, AWCS-anterior wall cavernous sinus, PCFd-posterior cranial fossa dura, BA-basilar artery, ICAc-cavernous portion of the internal carotid artery, P1-first segment of the posterior cerebral artery, PG-pituitary gland, Illcn-oculomotor nerve, PS-pituitary stalk, PL-pituitary ligament, SHA-superior hypophyseal artery, OC-optic chiasm, SCA-superior cerebellar artery.

dom of movement.

We maintain that also our proposal, like the previous ones $(9,10)$, takes advantage of another portal for the robotic arms to achieve an improved position of the arms, but all these solutions sacrifice the minimally invasive nature of the procedure itself. However, this is not significantly different from what is observed in robotic transaddominal-transthoracic procedures where different portals are used to gain a greater maneuverability and efficacy. Other transoral palatal sparing solutions for skull base robotic dissection have been described. But in this setting, the superior limit of the dissection is located more or less at the level of the midclivus ${ }^{(13)}$.

From a technical viewpoint, by a combined transnasal transcervical approach, it is possible to dissect the pituitary, the chias- matic and clival regions. The pituitary vascularization is correctly visualized as well as the basilar tip region. It is also possible to transpose the pituitary gland by cutting the meningohypophyseal trunk bilaterally and thus exposing the posterior portion of the Willis' circle perfectly. Furthermore, the oculomotor nerves are clearly identifiable between the posterior cerebral artery and the superior cerebellar artery. By rotating the lens laterally, the abducens nerves also come into view. On the contrary, in our hands, with a purely transoral route, we were able to manage only the inferior part of the clivus. We strongly underline that it was possible to expose all these structures even with the current instrumentation. The refinement in surgical instrumentation will offer greater possibilities in the future. The implementation of instruments for bony work will definitely increase the applicability of such a system in the forthcoming years. Among the drawbacks of this new technology, we must point out the absence 

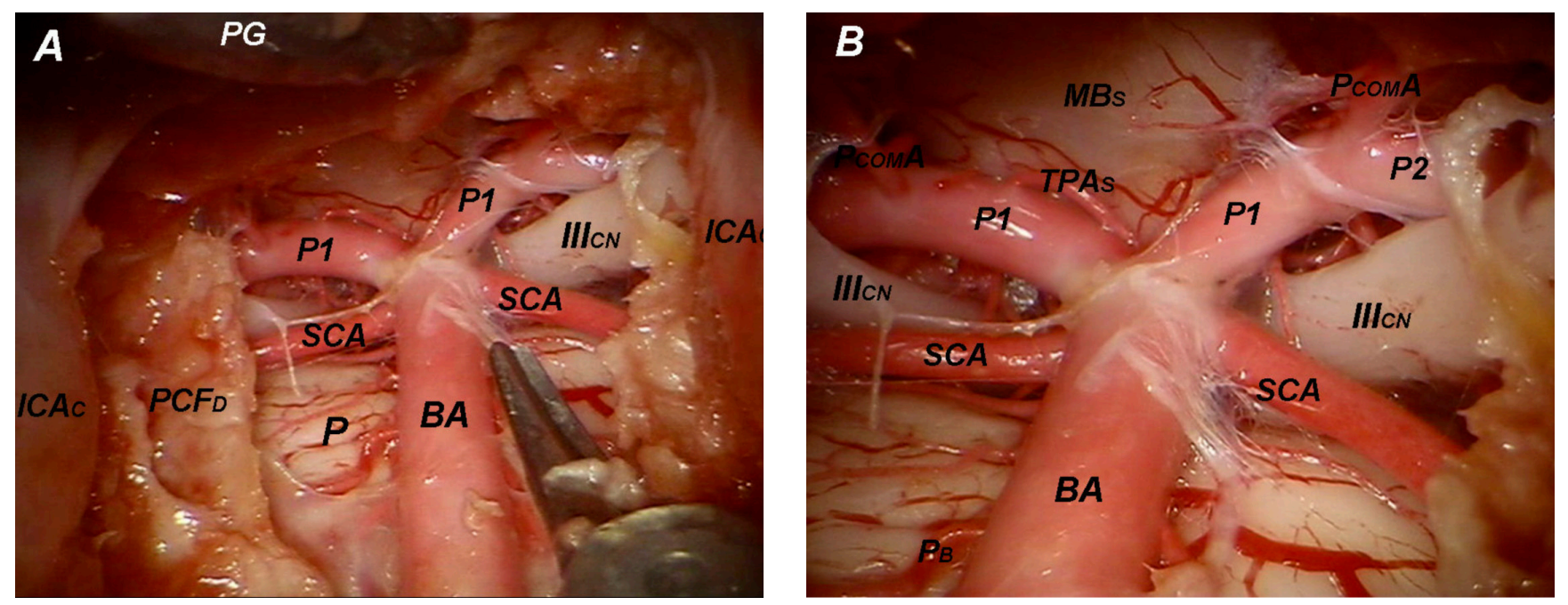

Figure 5. A and B. Basilar tip region exposition after pituitary transposition. Vision obtained using a $10 \mathrm{~mm}, 30^{\circ} \mathrm{scope}$, upward faced. A: Panoramic view. PCFd-posterior cranial fossa dura, P1-first segment of the posterior cerebral artery, Illcn-oculomotor nerve, ICAc-cavernous portion of the internal carotid artery, P-pons, PG-pituitary gland, SCA-superior cerebellar artery, BA-basilar artery. B: close view. BA-basilar artery, MBs-mammillary bodies, Illcn-oculomotor nerve, P1-first segment of the posterior cerebral artery, P2-second segment of the posterior cerebral artery, SCA-superior cerebellar artery, PcomA-posterior communicating artery, TPAs-talamoperforating arteries, Pb-pontine branch.

of tactile information. This is an important limitation of the da Vinci system especially in some regions and this problem will be addressed in the future. Another limit in the use of the da Vinci system in the skull base, and more in general in corridor surgery, is related to the fact that the robotic arms need to be aligned at a $90^{\circ}$ angle to one another to avoid physical interference with the camera and the surgical arms. But this geometrical necessity makes posterior cranial fossa dissection complex given the possible conflict between the arms. The intercarotic space at the level of the clival region represents a critical factor that impact the ability to dissect posterior cranial fossa and pituitary regions. A wide bone corridor is mandatory, and the more space is gained, the easier and more delicate the dissection is. A short, intercarotic distance makes dissection work more complex and increases the conflict between the instruments and robotic arms. Others have focused on the actual role and current limitations of the da Vinci surgical system robot in transoral management of the skull base ${ }^{(13)}$. In this sense, we strongly underline that a fully robotic skull base surgery will require the development of new tools for the da Vinci robotic arms. We underline, like others ${ }^{(24)}$, that a strict collaboration with the company is mandatory. Fortunately, the newer instruments seem to have significant advantages over the traditional ones in improving the ability to work in the upper regions.

We are strongly convinced that the ability to perform precise, tremor-free, bimanual surgery in confined cavities with instrumentation that exceeds the capabilities of the human hands ${ }^{(11)}$

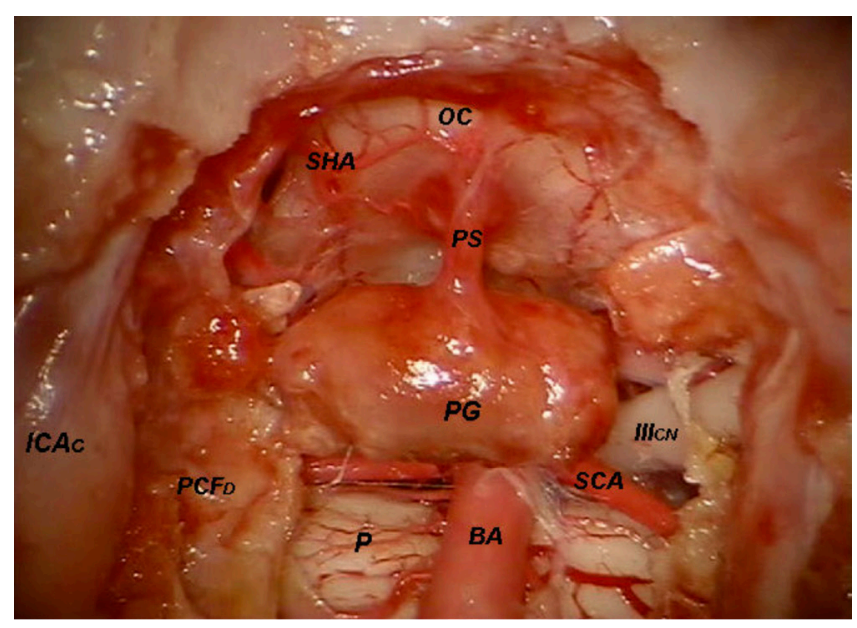

Figure 6. Sellar and suprasellar region: the superior hypophyseal artery and its arborization is clearly visible. Vision obtained using a $10 \mathrm{~mm}, 30^{\circ}$ scope, upward faced. PG-pituitary gland, PCFd-posterior cranial fossa dura, BA-basilar artery, ICAC-cavernous portion of the internal carotid artery, SCA-superior cerebellar artery, Illcn-oculomotor nerve, P-pons, PS-pituitary stalk, SHA-superior hypophyseal artery, OC-optic chiasm. 
truly represents a great opportunity for the patients of tomorrow.

\section{Conclusion}

Robotic assisted dissection of the skull base is feasible. Different solutions have been proposed. The placement of the optic system through the nose seem to offer a really significant advantage to this group of procedures.

The unique advantages of robotic surgery will prompt otolaryngologists and neurosurgeons to further refine and perfect its use and application in the complex field of skull base procedures.

\section{Acknowledgments}

We thank Mrs. Diana Elizabeth Hearn for her help in translation and revising the manuscript.

\section{Author contributions}

ID, CV, PC, FM, MT: substantial contribution to conception and design, acquisition, analysis, interpretation of data and final approval; VS, PB: drafting of article and critical revision for important intellectual content.

\section{Conflict of interest statement}

We state that all authors have seen and approved the manuscript 'Combined transnasal transcervical robotic dissection of posterior skull base: our experience', that there is no conflict of interest, that neither the submitted paper nor any similar paper, in whole or in part, other than an abstract or preliminary communication, has been or will be submitted to or published in any other printed or digital publication. We declare that all authors had full access to all the data in the study and take responsibility for the integrity of the data and the accuracy of the data analysis.

\section{References}

1. Jho HD, Ha HG. Endoscopic endonasal skull base surgery: Part 3 - The clivus and posterior fossa. Minim Invasive Neurosurg. 2004; 47: 16-23.

2. Solares CA, Fakhri S, Batra PS, Lee J, Lanza DC. Transnasal Endoscopic Resection of Lesions of the Clivus: A preliminary report. Laryngoscope. 2005; 115: 1917-1922.

3. Stamm AC, Pignatari SS, Vellutini E. Transnasal endoscopic surgical approaches to the clivus. Otolaryngol Clin North Am. 2006; 39: 639-656.

4. Frank G, Sciarretta V, Calbucci F, Farneti G, Mazzatenta D, Pasquini E. The endoscopic transnasal transsphenoidal approach for the treatment of cranial base chordomas and chondrosarcomas. Neurosurgery. 2006; 59: ONS50-7.

5. Fraser JF, Nyquist GG, Moore N, Anand VK, Schwartz TH. Endoscopic endonasal minimal access approach to the clivus: case series and technical nuances. Neurosurgery. 2010; 67: ONS150-8.

6. Holzmann D, Reisch R, Krayenbuhl N, Hug E, Bernays RL. The transnasal transclival approach for clivus chordoma. Minim Invasive Neurosurg. 2010; 53: 211-217

7. Lee JY, Lega B, Bhowmich D, et al. Da Vinci Robot-Assisted Transoral Odontoidectomy for Basilar Invagination. ORL J Otorhinolaryngol Relat Spec 2010; 72: 91-95

8. O'Malley BW jr, Quon H, Leonhardt FD, Chalian AA, Weinstein GS. Transoral robotic surgery for parapharyngeal space tumors. ORL J Otorhinolaryngol Relat Spec 2010; 72: 332-336.

9. Hanna EY, Holsinger C, DeMonte F, Kupferman M. Robotic endoscopic surgery of the skull base. A novel surgical approach. Arch Otolaryngol Head Neck Surg. 2007; 133: 1209-1214
10. O'Malley BW jr, Weinstein GS. Robotic anterior and midline skull base surgery: preclinical investigation. Int J Radiat Oncol Biol Phys. 2007; 69: S125-128.

11. Kupferman M, DeMonte F, Holsinger FC, Hanna E. Transantral robotic access to the pituitary gland. Otolaryngol Head Neck Surg. 2009; 141: 413-415.

12. Lee JY, O'Malley BW jr, Newman JG, et al. Transoral robotic surgery of craniocervical junction and atlantoaxial spine: a cadaveric study. J Neurosurg Spine. 2010; 12: 13-18.

13. Lee JY, O'Malley BW jr, Newman JG, et al. Transoral robotic surgery of the skull base: a cadaver and feasibility study. ORL J Otorhinolaryngol Relat Spec. 2010; 72: 181187.

14. Dallan I, Castelnuovo P, Montevecchi F, et al. Combined transoral transnasal roboticassisted nasopharyngectomy: a cadaveric feasibiity study. Eur Arch Otorhinolaryngol 2011 Mar 18 (epub ahead of print) PMID 21416233; DOI 10.1007/s00405-011-1550-x

15. Hadad G, Bassagasteguy L, Carrau RL, et al. A novel reconstructive technique after endoscopic expanded endonasal approaches: vascular pedicle nasoseptal flap. Laryngoscope. 2006; 116: 1882-1886.

16. Kassam AB, Thomas A, Carrau RL, et al. Endoscopic reconstruction of the cranial base using a pedicle nasoseptal flap. Neurosurgery. 2008; 63: ONS44-ONS53.

17. Lenz LZ, Brown S, Anand VK, Schwartz TH. 'Gasket-seal' watertight closure in minimalaccess endoscopic cranial base surgery. Neurosurgery. 2008; 62: ONSE342-3.

18. Harvey RJ, Nogueira JF jr, Schlosser RJ, Patel SJ, Vellutini E, Stamm A. Closure of large skull base defects after endoscopic transnasal craniotomy. J Neurosurg. 2009; 111: 371-379.

19. Bhatki AM, Pant $H$, Snyderman $\mathrm{CH}$, et al. Reconstruction of the cranial base after endonasal skull base surgery: Local Tissue Flaps. Op Tech Otolaryngol. 2010; 21: 74-82.

20. Bhatki AM, Pant $\mathrm{H}$, Snyderman $\mathrm{CH}$, et al. Reconstruction of the cranial base after endonasal skull base surgery: Regional Tissue Flaps. Op Tech Otolaryngol. 2010; 21: 83-90.

21. Kupferman ME, DeMonte F, Levine N, Hanna E. Feasibility of a Robotic Surgical Approach to Reconstruct the Skull Base. Skull Base. 2011; 21: 79-82.

22. Castelnuovo P, Dallan I, Battaglia P, Bignami M. Endoscopic endonasal skull base surgery: past, present and future. Eur Arch Otorhinolaryngol. 2010; 267: 649-663.

23. Vicini C, Dallan I, Canzi P, et al. Transoral robotic surgery of the tongue base in obstructive sleep Apnea-Hypopnea syndrome: Anatomic considerations and clinical experience. Head Neck 2011; DOI: 10.1002/ hed.21691.

24. Ponnusamy K, Chewning S, Mohr C. Robotic approaches to the posterior spine. Spine (Phila Pa 1976) 2009; 34: 2104-2109.

lacopo Dallan, M.D.

Otorhinolaryngologic Unit, University of Insubria

Viale Borri, 57

Varese, 21100

Italy

Tel: +39-0332-278426

Fax: +39-0332-278945

E-mail: iacopodallan@tiscali.it 